\title{
Mechanical Resonators for Quantum Optomechanics Experiments at Room Temperature
}

\author{
R. A. Norte, J. P. Moura, and S. Gröblacher* \\ Kavli Institute of Nanoscience, Delft University of Technology, Lorentzweg 1, 2628CJ Delft, The Netherlands
}

(Received 18 November 2015; published 5 April 2016)

\begin{abstract}
All quantum optomechanics experiments to date operate at cryogenic temperatures, imposing severe technical challenges and fundamental constraints. Here, we present a novel design of on-chip mechanical resonators which exhibit fundamental modes with frequencies $f$ and mechanical quality factors $Q_{\mathrm{m}}$ sufficient to enter the optomechanical quantum regime at room temperature. We overcome previous limitations by designing ultrathin, high-stress silicon nitride $\left(\mathrm{Si}_{3} \mathrm{~N}_{4}\right)$ membranes, with tensile stress in the resonators' clamps close to the ultimate yield strength of the material. By patterning a photonic crystal on the SiN membranes, we observe reflectivities greater than $99 \%$. These on-chip resonators have remarkably low mechanical dissipation, with $Q_{\mathrm{m}} \sim 10^{8}$, while at the same time exhibiting large reflectivities. This makes them a unique platform for experiments towards the observation of massive quantum behavior at room temperature.
\end{abstract}

DOI: 10.1103/PhysRevLett.116.147202

Optomechanical systems, where light is coupled to mechanical motion via the radiation pressure force, have generated enormous interest over the past years. They are promising candidates for testing macroscopic quantum physics, have great potential as quantum transducers between distinct quantum systems and have unique capabilities for sensing applications [1]. State-of-the-art systems have recently demonstrated ground state cooling [2,3], mechanical quantum state preparation [4,5], entanglement [6], and squeezing of both the optical [7,8] as well as the mechanical mode [9-11]. Experiments involving such optomechanical systems in the quantum regime are technically very challenging and so far have exclusively operated at cryogenic temperatures. This poses serious restrictions on the type of experiments that are feasible. Without the need for cryogenic precooling, one could envision their use as hybrid quantum systems with, for example, atomic gases [12] or single atoms [13]. It would also open up practical avenues for real world applications of such quantum optomechanical systems.

One of the most successful implementation of mechanical oscillators for such (quantum) optomechanics experiments are devices made of high-stress silicon nitride $\left(\mathrm{Si}_{3} \mathrm{~N}_{4}\right)$, which have been utilized in quantum-limited accelerometers [14], coupling of their motion to ultracold atoms [15,16], optomechanics in 3D microwave cavities [17], microwave-to-optical wavelength conversion [18], and quadratic coupling in cavity optomechanics [19].

However, even these low mechanical dissipation oscillators have to date not operated in a regime where realistic quantum experiments at room temperature are feasible. The benchmark for this elusive regime is the $f \times Q_{\mathrm{m}}$ product of the resonator which requires the mechanical quality factor $Q_{\mathrm{m}}$ to be larger than the number of thermal phonons at room temperature $\left(f \times Q_{\mathrm{m}}>k_{\mathrm{B}} T_{\text {room }} / h\right)$, with $h$ being the Planck and $k_{\mathrm{B}}$ the Boltzmann constant [20]. This regime will allow for ground state cooling using the radiation pressure force and hence, for experiments operating in the quantum domain. In general, the quality factor-frequency product also determines the number of coherent oscillations the resonator can undergo before one phonon enters the system; i.e., $N_{\mathrm{osc}}=Q_{\mathrm{m}} f \cdot h / 2 \pi k_{\mathrm{B}} T$. Experimental realizations of onchip mechanical resonators that exceed this requirement have all been demonstrated in higher-order mechanical modes [21,22]. Such mechanical systems are however in practice not useful for cooling experiments as higher order modes are enveloped by numerous neighboring modes, which increase the displacement background noise as one cools the mode of interest. To avoid this limit, it is important to couple to the fundamental mode of the resonator. An additional challenge is to operate at mechanical frequencies beyond $10^{5} \mathrm{~Hz}$, where commercial lasers exhibit a minimal amount of classical noise and can relatively easily be quantum limited to shot noise in order to avoid heating or decoherence of the mechanics through noise [23]. Another difficulty for realistic quantum optomechanics experiments at room temperature is that often good mechanical quality is mutually exclusive with good optical reflectivity [24-26]. This limits the achievable coupling rates and increases the necessary optical power to a level where absorption potentially becomes a practical limitation for cooling and quantum experiments.

In this Letter, we demonstrate the first optomechanical platform that overcomes all these limitations, paving the way for room temperature experiments in the quantum regime. We fabricate on-chip optomechanical mirrors which exhibit $f \times Q_{\mathrm{m}}$ products of their fundamental mechanical modes above the requirement for ground state cooling without cryogenics (Fig. 1). With a center-of-mass frequency of $\sim 150 \mathrm{kHz}$ and mechanical quality factor $Q_{\mathrm{m}} \sim 10^{8}$, this new generation of $\mathrm{Si}_{3} \mathrm{~N}_{4}$ tethered membranes are on par with the state of the art in optically levitated nanospheres, known for their extreme mechanical isolation and ultralow dissipation, which are only limited by gas-molecule collisions in high vacuum and photon 

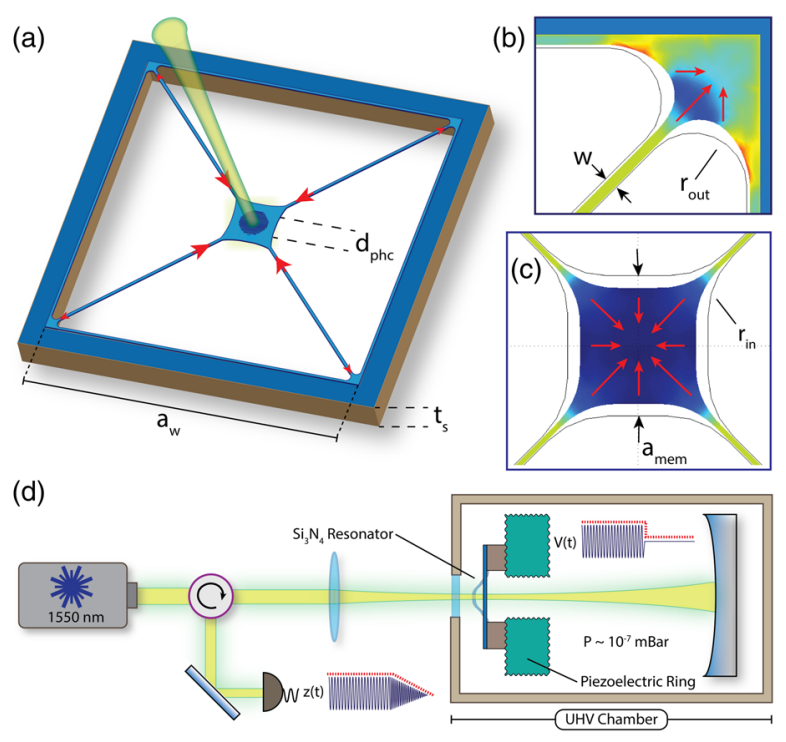

FIG. 1. (a) Schematic of our tethered membrane which consists of a central membrane connected to the silicon substrate through a series of thin tethers. A central photonic crystal reflector can be used for increased reflectivities. Shown are our design parameters which are individually swept keeping all others constant to observe their effect on the $f \times Q_{\mathrm{m}}$ product. Finite element simulations map out the strain throughout the resonator and calculate deformation due to the relaxation of the material at the (b) tether clamps and (c) central membrane, which together significantly enhance the tensile stress in the tethers. (d) Schematic of ringdown measurement setup. The membrane is resonantly driven by a piezoelectric transducer inside an optical interferometer. We determine the mechanical dissipation by observing the ringdown of the mechanical resonance (see text for details).

recoil heating $[27,28]$. In order to achieve such remarkably low dissipation rates of $\Gamma_{\mathrm{m}} / 2 \pi=f / Q_{\mathrm{m}} \approx 1.4 \mathrm{mHz}$ with a tethered system, we design ultrathin high-stress $\mathrm{Si}_{3} \mathrm{~N}_{4}$ membranes which enhance the intrinsic stress in crucial tether regions-significantly reducing clamping and bending losses [29]. A key observation is that high-stress membranes have mechanical frequencies which are stress dominated, meaning that one can minimize the thickness of the resonator in order to reduce bending losses without significantly reducing the mechanical mode frequencies. We fabricate tethered membranes with ultralow dissipation rates by engineering up to $6 \mathrm{GPa}$ of stress within films as thin as $15 \mathrm{~nm}$ and intrinsic stress of $1.3 \mathrm{GPa}$. Using finite element simulations to calculate the stress throughout the resonators, we push the tensile stress in the resonator's clamps to values near the ultimate yield strength of low-pressure chemical vapor deposition (LPCVD) $\mathrm{Si}_{3} \mathrm{~N}_{4}$. In addition, we are able to show that we can increase the intrinsic optical reflectivity $R$ of these membranes to up to $99.3 \%$ by patterning a two-dimensional photonic crystal onto the structure. These devices enable coupling rates that allow ground state cooling from room temperature with realistic parameters in a membrane-in-the-middle design
[30-33] and can also be used as an end mirror of a Fabry-Pérot cavity [25,34-36].

The central mirror on the tethered membranes is a $2 \mathrm{D}$ photonic crystal device, that is designed using finite difference time domain (FDTD) simulations. They are similar to previous designs of grating reflectors [37] and photonic crystals $(\mathrm{PhC})[38,39]$, which usually consist of an array of either lines or holes etched into the dielectric, respectively. Such a periodic change in the refractive index allows for a band gap to be tailored for a specific wavelength, resulting in (simulated) reflectivities $>99.9 \%$. Experiments to date employing such photonic crystal mirrors for optomechanical resonators have however suffered from a trade-off between high reflectivity and high-mechanical quality, due to the requirement of thick dielectrics in order to achieve large $R$ [39]. We confirm this by measuring $\mathrm{PhC}$ mirrors consisting of a square lattice of holes as a function of silicon nitride thickness (see Supplemental Material [40]). In order to circumvent this design issue, we follow two slightly different approaches: we either leave a thick cylindrical slab of $\mathrm{SiN}$ around the $\mathrm{PhC}$ [see Fig. 2(c)] or instead of using holes, we use pillars, resulting in an inverse photonic crystal [see Fig. 2(d)]. While the latter design still suffers from fabrication imperfections resulting in reflectivities of $\sim 95 \%$, the other new design allows us to reach $R>99 \%$ (Fig. 2). We show (a)

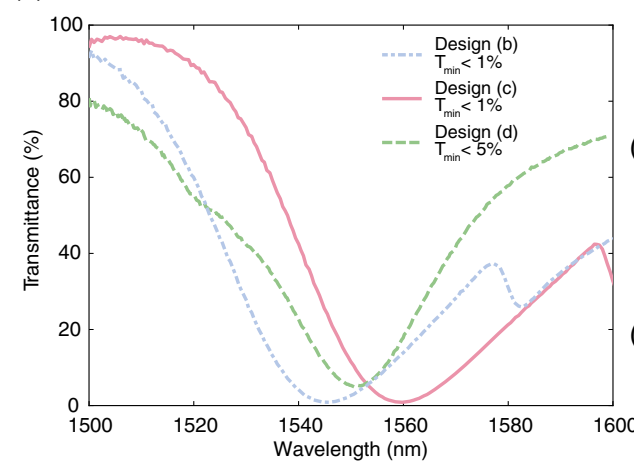

(b)

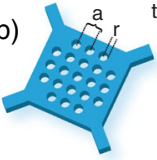

(c)

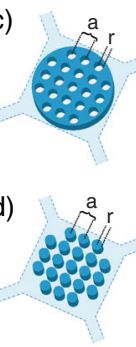

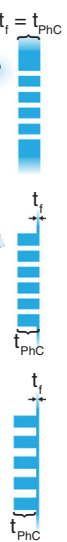

FIG. 2. (a) The measured transmittance $T$ for three different designs of tethered mechanical membranes (b)-(d). The thickness of the photonic crystal $t_{\mathrm{PhC}}$ was chosen to be $200 \mathrm{~nm}$ for all designs. The maximum reflectivity $\left(1-T_{\min }\right)$ strongly depends on the thickness of the silicon nitride (see Supplemental Material [40]). For (b), this is in competition with the requirement of thin films (i.e., small $t_{\mathrm{f}}$ ) for good mechanical quality (cf. Fig. 3). We overcome this limitation by either leaving a thick cylindrical slab in the center of the membrane (c) or by using an inverse photonic crystal design (d). These designs decouple the mechanical and optical properties of the membrane and show similar optical performances to (b). We use a simple transmittance measurement to infer reflectivity. We experimentally confirm this by using a PhC membrane as one end mirror of a Fabry-Pérot cavity in combination with a second mirror with known reflectivity and measuring the finesse. 
that both methods decouple the mechanical from the optical properties and allow for optomechanical devices with simultaneous high $Q_{\mathrm{m}}$ and high $R$.

We also study the tethered membranes' mechanical parameters, several of which suffer from the conventional trade-off between either good mechanical quality factor or high frequency (see Fig. 1 and Table IV, Supplemental Material [40]). For example, extending the length of the tethers by increasing the size of the window $a_{\mathrm{w}}$ in order to reduce clamping and bending losses not only results in higher mechanical quality factors but also lower frequencies [41]. Achieving ultrahigh $Q_{\mathrm{m}}$ with this parameter will result in very low fundamental modes, where lowfrequency classical noise in lasers becomes a compelling limitation on radiation-pressure sideband cooling (for more detail and discussion on design parameters, see the Supplemental Material [40]).

It is known that for fundamental modes of long, thin nanostrings (width, $w \approx 3-5 \mu \mathrm{m}$ ), mechanical dissipation starts to be dominated by bending losses [42]. By accounting for the dominating effect of flexural bending near the clamps of tensile strings, one can express the quality factor for the fundamental mode of doubly clamped strings as

$Q_{\text {str }}=\left[\frac{\pi^{2}}{12} \frac{E}{\sigma}\left(\frac{t_{\mathrm{f}}}{L}\right)^{2}+1.0887 \sqrt{\frac{E}{\sigma}}\left(\frac{t_{\mathrm{f}}}{L}\right)\right]^{-1} Q_{\text {bending }}$,

where $E$ is the $\mathrm{Si}_{3} \mathrm{~N}_{4}$ Young's modulus, $\sigma$ is the stress in the string, $t_{\mathrm{f}}$ is the film thickness, $L$ is the length of the nanostring, and $Q_{\text {bending }}$ is the quality factor due to bending losses in a relaxed string, which is mostly dependent on intrinsic material damping. From Eq. (1), one finds that the quality factor of a string can be enhanced by using thinner strings with decreasing $t_{\mathrm{f}}$. A crucial observation from thin plate theory is that membranes under large tensile forces have stress-dominated mechanical frequencies [i.e., $f_{11}=1 /(\sqrt{2} L) \sqrt{\sigma / \rho}$, where $\rho$ is the material's density] which are independent of membrane thickness. Since our devices' dissipation is dominated by bending losses through the tethers and have mechanical modes which are independent of thickness, we can engineer thinner membranes with increased mechanical quality factors and negligible impact on the frequency. This design scheme overcomes the trade-off between $Q_{\mathrm{m}}$ and $f$ in order to realize optomechanical resonators with unprecedented enhancement of the fundamental mode $f \times Q_{\mathrm{m}}$ product.

In addition, substrate thickness plays an important role in anchoring losses for out-of-plane fundamental modes, where larger vibrational displacements of thinner substrates near the clamping points of a fundamental mode significantly increase mechanical dissipation [43]. Previous studies found some enhancement in 30-50 nm thick square membranes when moving from $200 \mu \mathrm{m}$ substrates to $500 \mu \mathrm{m}$ with a negligible effect in thicker $\mathrm{Si}_{3} \mathrm{~N}_{4}$ films $\left(t_{\mathrm{f}} \geq 100 \mathrm{~nm}\right)$ [44]. Silicon substrate thickness and silicon nitride film thickness are the parameters we focus on to achieve significant enhancements in $f \times Q_{\mathrm{m}}$. We investigate these effects by fabricating our resonators from ultrathin films $\geq 15 \mathrm{~nm}$ and on substrates as thick as $900 \mu \mathrm{m}$ (for fabrication details see the Supplemental Material [40]).

We determine the mechanical quality factors of our membranes by performing ringdown measurements using a piezoelectric stack in an optical interferometer [see Fig. 1(d)]. Due to viscous damping, which becomes increasingly dominant with thinner membranes, our measurements are conducted inside an ultrahigh vacuum chamber at $<10^{-7}$ mbar. Positioning stages are used to align the chip with the membranes to a $20 \mu \mathrm{m}$ spot of a $1550 \mathrm{~nm}$ laser. The chip is placed onto the piezolectric stack under its own weight since any type of clamping or gluing of the chip to the experimental setup can reduce the mechanical quality factor by several orders of magnitude [44]. Each of our chips has 9-16 resonators which allow us to collect several data points for each parameter sweep. We start with a nominal resonator design (see Supplemental Material [40] for details) and adjust each parameter keeping all others fixed. In Fig. 3, we plot the effects on $f \times Q_{\mathrm{m}}$ by varying the thickness of the silicon nitride films and silicon substrates for the nominal membrane design. We find that losses in thicker $\mathrm{Si}_{3} \mathrm{~N}_{4}$ resonators $\left(t_{\mathrm{f}} \geq 100 \mathrm{~nm}\right)$ are dominated by bending losses. From the data, we also see

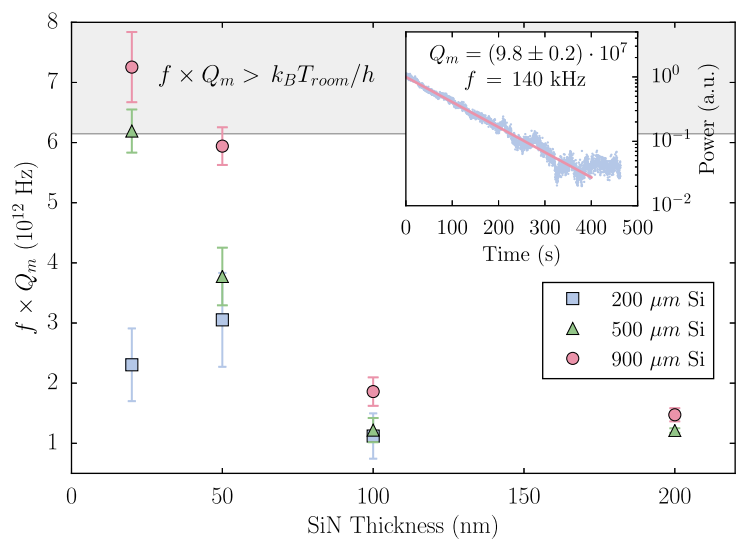

FIG. 3. Shown is the $f \times Q_{\mathrm{m}}$ product for the nominal design [40] of our tethered membrane for various silicon substrate $(\{200,500,900\} \mu \mathrm{m})$ and $\mathrm{Si}_{3} \mathrm{~N}_{4}$ film $(\{20,50,100,200\} \mathrm{nm})$ thicknesses. Each point is the average of measurements of resonators with identical geometry. In addition, we verified that the results are independent of the particular $\mathrm{PhC}$ design. For thin silicon substrates $\left(t_{\mathrm{s}}=200 \mu \mathrm{m}\right)$, the anchoring losses completely dominate and result in minimal enhancement of the mechanical quality factor even at $t_{\mathrm{f}}=20 \mathrm{~nm}$. However, these ultrathin films exhibit $f \times Q_{\mathrm{m}}$ products above the ground state cooling limit (shaded region) when fabricated on thicker substrates, where anchoring losses are less pronounced. The inset shows the ringdown measurement for the best observed value for a single device with $Q_{\mathrm{m}}=9.8 \pm 0.2 \times 10^{7}$ at $f=140 \mathrm{kHz}$ for a $20 \mathrm{~nm}$ thick film, with otherwise nominal parameters. 
that low dissipation in ultrathin resonators can be completely dominated by anchoring losses in thin substrates $(\sim 200 \mu \mathrm{m})$. A clear enhancement in the $f \times Q_{\mathrm{m}}$ product is observed, consistently exceeding the above requirement for ground state cooling at room temperatures as one fabricates thinner tethered membranes on increasingly thicker substrates, where anchoring losses are no longer a limiting factor on $Q_{\mathrm{m}}$.

In order to push the devices to their material limits, we engineer the stress at the clamps (the dominant source of flexural bending loss) to just short of the $\mathrm{Si}_{3} \mathrm{~N}_{4}$ ultimate yield strength $(\approx 6.4 \mathrm{GPa}[45])$. At thicknesses $\leq 20 \mathrm{~nm}$, the silicon nitride membranes become increasingly susceptible to plastic deformation when subjected to small viscous forces due to handling or large temperature variations during wet chemical processing. Figure 4(b) shows the necking that occurs when the resonator is subjected to these small forces which result in large frequency drops from $\sim 170 \mathrm{kHz}$ to $\sim 60 \mathrm{kHz}$ and reduction in $Q_{\mathrm{m}}$ to $\sim 10^{5}$. Necking is a form of irreversible plastic deformation that occurs when excessively large strains localize in small cross sections. In order to minimize such effects, we use polytetrafluoroethylene (PTFE) turbulence shielding holders [46] which gently dilute the resonators from one liquid to the next during fabrication. This significantly reduces the resonators' exposure to viscous forces and surface tension in the wet chemistry processes

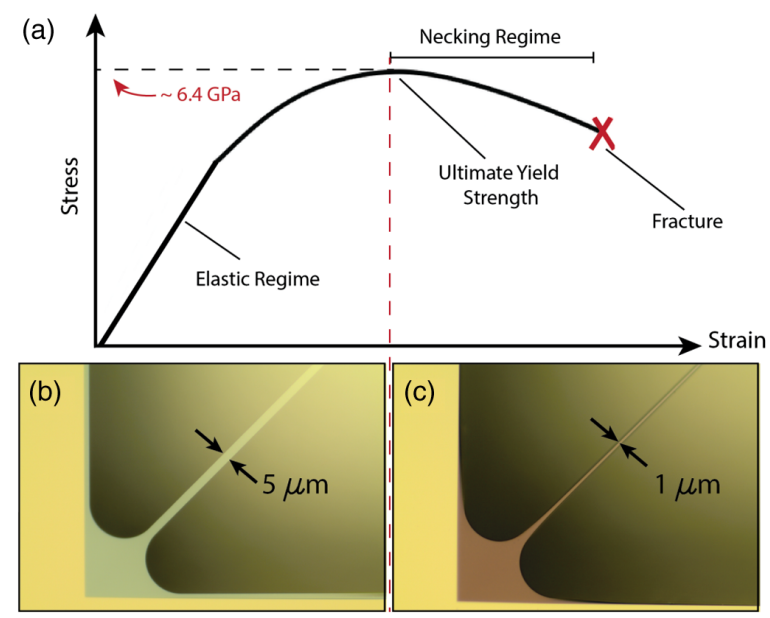

FIG. 4. (a) Sketch of the stress-strain curve for silicon nitride. We design our structures such that for the thinnest films, we operate in the regime close to the ultimate yield strength of the material. (b) Microscope image showing a nominal clamp with outer fillet $r_{\text {out }}=20 \mu \mathrm{m}$ and tether width $w=5 \mu \mathrm{m}$ when properly fabricated with turbulence reducing holders. (c) shows typical plastic deformation in very thin films $(\leq 20 \mathrm{~nm})$. This occurs at the tether clamps where the stress is the largest and when the resonators are being exposed to small viscous forces or temperature gradients during fabrication. The tether is deformed to a width of $\sim 1 \mu \mathrm{m}$. required to release, clean, and rinse the sample thoroughly [46]. At thicknesses below $20 \mathrm{~nm}$, we observe consistently reduced $Q_{\mathrm{m}} \sim 10^{7}$. Ultrathin films below $20 \mathrm{~nm}$ produce delicate structures which make it difficult to attribute the lower quality factors to intrinsic limits of the sensitive handling during fabrication or whether other surfacedominant loss mechanisms, such as Akhiezer damping, become more dominant loss channels. The inset in Fig. 3 shows a ringdown for our best device at $20 \mathrm{~nm}$ silicon nitride thickness, $900 \mu \mathrm{m} \mathrm{Si}$ wafer thickness, and mechanical quality factor $Q_{\mathrm{m}}=9.8 \pm 0.2 \times 10^{7}$ at a fundamental mode frequency of $140 \mathrm{kHz}\left(f \times Q_{\mathrm{m}}=1.37 \times 10^{13} \mathrm{~Hz}\right)$.

With the demonstrated combination of large reflectivities and low mechanical dissipation, we will be able to access the optomechanical quantum regime from room temperature. A first demonstration of such quantum behavior could be achieved by cooling the mechanical mode into its quantum ground state, which is already realistic with these device parameters (see Supplemental Material [40] for detailed calculations).

To conclude, we studied the dissipation mechanisms in a new regime of ultrathin, highly stressed optomechanical resonators and their effects on mechanical quality factor and fundamental mode frequency. By moving to these thin devices on thick silicon substrates and by engineering the stress in their tethers to near the ultimate yield strength of $\mathrm{Si}_{3} \mathrm{~N}_{4}$, we are able to overcome a well-known trade-off between frequency and mechanical quality factor to achieve fundamental modes $f \times Q_{\mathrm{m}}$, which are more than twice the requirement for quantum ground state cooling from room temperature. Our on-chip device performances are on par with the best values measured for optically levitated nanospheres without the need for high power trapping lasers or complex experimental setups. We also demonstrate the possibility to combine our resonators with different photonic crystal mirror designs which result in reflectivities between $95 \%$ and $99 \%$, while simultaneously achieving ultrahigh $Q_{\mathrm{m}}$. These results allow us to finally realize experiments to laser cool a mechanical oscillator from room temperature to its quantum ground state (see Supplemental Material [40]). Such reflective tethered membranes are also ideal for optical trapping configurations that enhance the frequency and the mechanical quality factor even further, while avoiding thermal bistabilities which become a severe limitation at high laser powers $[47,48]$. With a $Q_{\mathrm{m}} \sim 10^{8}$, our dissipation rates are only matched in other silicon nitride membranes with the use of cryogenic cooling near $14 \mathrm{mK}$, with an improvement of 2 orders of magnitude in mechanical quality factor from room temperature [49]. This allows one to speculate that coupling our new generation of resonators to such low temperature baths could yield $Q_{\mathrm{m}} \sim 1$ billion.

Our devices have the potential to allow for fundamental tests of quantum physics by generating massive, nonclassical states of a mechanical oscillator, for example, 
in space, where complicated cryogenic setups are not feasible [50]. In addition, thanks to the ultralow mechanical dissipation, it is possible to push boundaries of applications in ultrasensitive (e.g., force) detection [51-53], as has recently been demonstrated [54]. For the devices used here, we calculate a force sensitivity of about $10 \mathrm{aN} / \mathrm{Hz}^{1 / 2}$, which, together with Ref. [54] and to our best knowledge, is the highest to date at room temperature. Achieving this level of dissipation in an on-chip design heralds a realistic building block towards optically linked silicon-based quantum networks [55] operating at room temperature.

We would like to thank M. Aspelmeyer, L. Bavdaz, A. G. Krause, I. Marinković, and G. A. Steele for fruitful discussions and help with early measurements. We also acknowledge valuable support from the Kavli Nanolab Delft, in particular, from C. de Boer and M. Zuiddam. This project was supported by the European Research Council (ERC StG Strong-Q, Grant No. 676842) and by the Netherlands Organisation for Scientific Research (NWO/ OCW), as part of the Frontiers of Nanoscience program.

*s.groeblacher@tudelft.nl

[1] M. Aspelmeyer, T. J. Kippenberg, and F. Marquardt, Rev. Mod. Phys. 86, 1391 (2014).

[2] J. Chan, T. P. M. Alegre, A. H. Safavi-Naeini, J. T. Hill, A. Krause, S. Gröblacher, M. Aspelmeyer, and O. Painter, Nature (London) 478, 89 (2011).

[3] J. D. Teufel, T. Donner, D. Li, J. W. Harlow, M. S. Allman, K. Cicak, A. J. Sirois, J. D. Whittaker, K. W. Lehnert, and R. W. Simmonds, Nature (London) 475, 359 (2011).

[4] A. D. O'Connell, M. Hofheinz, M. Ansmann, R. C. Bialczak, M. Lenander, E. Lucero, M. Neeley, D. Sank, H. Wang, M. Weides et al., Nature (London) 464, 697 (2010).

[5] R. Riedinger, S. Hong, R. A. Norte, J. A. Slater, J. Shang, A. G. Krause, V. Anant, M. Aspelmeyer, and S. Gröblacher, Nature (London) 530, 313 (2016).

[6] T. Palomaki, J. Teufel, R. Simmonds, and K. Lehnert, Science 342, 710 (2013).

[7] A. H. Safavi-Naeini, S. Gröblacher, J. T. Hill, J. Chan, M. Aspelmeyer, and O. Painter, Nature (London) 500, 185 (2013).

[8] T. P. Purdy, P.-L. Yu, R. W. Peterson, N. S. Kampel, and C. A. Regal, Phys. Rev. X 3, 031012 (2013).

[9] E. E. Wollman, C. Lei, A. Weinstein, J. Suh, A. Kronwald, F. Marquardt, A. Clerk, and K. Schwab, Science 349, 952 (2015).

[10] J.-M. Pirkkalainen, E. Damskägg, M. Brandt, F. Massel, and M. A. Sillanpää, Phys. Rev. Lett. 115, 243601 (2015).

[11] F. Lecocq, J. B. Clark, R. W. Simmonds, J. Aumentado, and J. D. Teufel, Phys. Rev. X 5, 041037 (2015).

[12] P. Treutlein, D. Hunger, S. Camerer, T. W. Hänsch, and J. Reichel, Phys. Rev. Lett. 99, 140403 (2007).

[13] K. Hammerer, M. Wallquist, C. Genes, M. Ludwig, F. Marquardt, P. Treutlein, P. Zoller, J. Ye, and H. J. Kimble, Phys. Rev. Lett. 103, 063005 (2009).
[14] A. G. Krause, M. Winger, T. D. Blasius, Q. Lin, and O. Painter, Nat. Photonics 6, 768 (2012).

[15] A. Jöckel, A. Faber, T. Kampschulte, M. Korppi, M. T. Rakher, and P. Treutlein, Nat. Nanotechnol. 10, 55 (2014).

[16] D. M. Stamper-Kurn, in Cavity Optomechanics, edited by M. Aspelmeyer et al. (Springer, Heidelberg Berlin, 2014), Chap. (13), pp. 283-325.

[17] M. Yuan, V. Singh, Y. M. Blanter, and G. A. Steele, Nat. Commun. 6, 8491 (2015).

[18] R. Andrews, R. Peterson, T. Purdy, K. Cicak, R. Simmonds, C. Regal, and K. Lehnert, Nat. Phys. 10, 321 (2014).

[19] J. Thompson, B. Zwickl, A. Jayich, F. Marquardt, S. Girvin, and J. Harris, Nature (London) 452, 72 (2008).

[20] F. Marquardt, J. P. Chen, A. A. Clerk, and S. M. Girvin, Phys. Rev. Lett. 99, 093902 (2007).

[21] D. J. Wilson, C. A. Regal, S. B. Papp, and H. J. Kimble, Phys. Rev. Lett. 103, 207204 (2009).

[22] S. Chakram, Y. S. Patil, L. Chang, and M. Vengalattore, Phys. Rev. Lett. 112, 127201 (2014).

[23] A. H. Safavi-Naeini, J. Chan, J. T. Hill, S. Gröblacher, H. Miao, Y. Chen, M. Aspelmeyer, and O. Painter, New J. Phys. 15, 035007 (2013).

[24] G. M. Harry, A. M. Gretarsson, P. R. Saulson, S. E. Kittelberger, S. D. Penn, W. J. Startin, S. Rowan, M. M. Fejer, D. R. M. Crooks, G. Cagnoli et al., Classical Quantum Gravity 19, 897 (2002).

[25] S. Gröblacher, J. B. Hertzberg, M. R. Vanner, S. Gigan, K. C. Schwab, and M. Aspelmeyer, Nat. Phys. 5, 485 (2009).

[26] D. Kleckner, B. Pepper, E. Jeffrey, P. Sonin, S. M. Thon, and D. Bouwmeester, Opt. Express 19, 19708 (2011).

[27] D. E. Chang, C. A. Regal, S. B. Papp, D. J. Wilson, J. Ye, O. Painter, H. J. Kimble, and P. Zoller, Proc. Natl. Acad. Sci. U.S.A. 107, 1005 (2009).

[28] J. Gieseler, L. Novotny, and R. Quidant, Nat. Phys. 9, 806 (2013).

[29] R. Zhang, C. Ti, M. I. Davano, Y. Ren, V. Aksyuk, Y. Liu, and K. Srinivasan, Appl. Phys. Lett. 107, 131110 (2015).

[30] A. Nunnenkamp, K. Børkje, J. Harris, and S. Girvin, Phys. Rev. A 82, 021806 (2010).

[31] Z. J. Deng, Y. Li, M. Gao, and C. W. Wu, Phys. Rev. A 85, 025804 (2012).

[32] K. Usami, A. Naesby, T. Bagci, B. M. Nielsen, J. Liu, S. Stobbe, P. Lodahl, and E. S. Polzik, Nat. Phys. 8, 168 (2012).

[33] A. Jayich, J. Sankey, B. Zwickl, C. Yang, J. Thompson, S. Girvin, A. Clerk, F. Marquardt, and J. Harris, New J. Phys. 10, 095008 (2008).

[34] S. Gigan, H. R. Böhm, M. Paternostro, F. Blaser, G. Langer, J. B. Hertzberg, K. C. Schwab, D. Bäuerle, M. Aspelmeyer, and A. Zeilinger, Nature (London) 444, 67 (2006).

[35] O. Arcizet, P.-F. Cohadon, T. Briant, M. Pinard, and A. Heidmann, Nature (London) 444, 71 (2006).

[36] A. Schliesser, P. Del'Haye, N. Nooshi, K. J. Vahala, and T. J. Kippenberg, Phys. Rev. Lett. 97, 243905 (2006).

[37] C. Stambaugh, H. Xu, U. Kemiktarak, J. Taylor, and J. Lawall, Ann. Phys. (Amsterdam) 527, 81 (2015). 
[38] C. H. Bui, J. Zheng, S. W. Hoch, L. Y. T. Lee, J. G. E. Harris, and C. W. Wong, Appl. Phys. Lett. 100, 021110 (2012).

[39] K. Makles, T. Antoni, A. G. Kuhn, S. Deléglise, T. Briant, P.-F. Cohadon, R. Braive, G. Beaudoin, L. Pinard, C. Michel et al., Opt. Lett. 40, 174 (2015).

[40] See Supplemental Material at http://link.aps.org/ supplemental/10.1103/PhysRevLett.116.147202 for additional calculations and detailed measurements for various design parameters of the membranes.

[41] S. S. Verbridge, R. Ilic, H. G. Craighead, and J. M. Parpia, Appl. Phys. Lett. 93, 013101 (2008).

[42] S. Schmid, K. D. Jensen, K. H. Nielsen, and A. Boisen, Phys. Rev. B 84, 165307 (2011).

[43] A. Darvishian, B. Shiari, G. He, and K. Najafi, in 2015 IEEE International Symposium on Inertial Sensors and Systems (ISISS) (IEEE, New York, 2015), pp. 1-4.

[44] D. J. Wilson, Ph.D. thesis, California Institute of Technology, 2012.

[45] A. Kaushik, H. Kahn, and A. H. Heuer, J. Microelectromech. Syst. 14, 359 (2005).
[46] R. A. Norte, Ph.D. thesis, California Institute of Technology, 2014.

[47] M. Bhattacharya and P. Meystre, Phys. Rev. Lett. 99, 073601 (2007).

[48] K.-K. Ni, R. Norte, D. J. Wilson, J. D. Hood, D. E. Chang, O. Painter, and H. J. Kimble, Phys. Rev. Lett. 108, 214302 (2012).

[49] M. Yuan, M. A. Cohen, and G. Steele, Appl. Phys. Lett. 107, 263501 (2015).

[50] A. Pilan-Zanoni, J. Burkhardt, U. Johann, M. Aspelmeyer, R. Kaltenbaek, and G. Hechenblaikner, arXiv:1508.01032.

[51] D. Rugar, R. Budakian, H. J. Mamin, and B. W. Chui, Nature (London) 430, 329 (2004).

[52] Y. Tao, J. M. Boss, B. A. Moores, and C. L. Degen, Nat. Commun. 5, 3638 (2014).

[53] A. Vinante, M. Bahrami, A. Bassi, O. Usenko, G. Wijts, and T. Oosterkamp, Phys. Rev. Lett. 116, 090402 (2016).

[54] C. Reinhardt, T. Müller, A. Bourassa, and J. C. Sankey, Phys. Rev. X 6, 021001 (2016).

[55] H. J. Kimble, Nature (London) 453, 1023 (2008). 